\title{
The tri-harmonic plate bending equation
}

\author{
I. D. Kotchergenko \\ Kotchergenko Engenharia Ltda., Brazil
}

\begin{abstract}
This is the third paper published at WIT Transactions on Modeling and Simulation focusing on the application of the areolar strain concept to revisit subjects of the theory of elasticity, allowing a deeper insight on some old topics. In Vol. 46, 2007, WIT Press, a new concept of strain is introduced which does not distinguish finite from infinitesimal strain. Besides the classical "forward" strain, it incorporates the "sidelong" strain into its imaginary part. Instead of comparing the change in distance between two contiguous points, this concept describes the complete state of strain in an areola that surrounds a given point. This strain is given by a total derivative, in agreement with its physical meaning. As a consequence, the relative displacement between two arbitrary points of the strained plane is obtained by a line integral of the strain, along any path joining these points. In Vol. 55, 2013, WIT Press, the nature of the propagation of plane shear waves is cleared up, showing that although a point of the plane performs lateral vibration only, the areola centered at this point pulses in the direction of the wave path, through a combination of rotation and shear. In the present paper, a similar behavior is explored to show that the rotation of the neutral planes of beams and plates does not follow the rotation of a point (areola) situated on these planes. A compatible field of strains is defined at the neighborhood of the neutral plane of the plate and then forced to comply with the boundary conditions. A sixth-order differential equation is obtained, which gives exactly the shearing forces, eliminating thus Kirchhoff's anomaly. This tri-harmonic equation allows the prescription of complete boundary conditions for plate bending analysis.
\end{abstract}

Keywords: areolar strain, beam shear, plate shear, tri-harmonic.

\section{Introduction}

Presenting a new equation for beam, and for plate bending and shear, looks an adventurous task. The deformation by shear of beams and plates is a long time 
subject of research by some of the most prominent scientists. These scientists however did not derived profit of a correct system of compatibility equations which considers rotation as part of the strain. This lack has turned elasticity a very complicate subject. The areolar strain concept was presented at Kotchergenko [912], with application to finite rotations, orthotropic materials, finite element models and wave propagation, but a summary review is here presented. The areolar strain approach does not distinguish finite from infinitesimal strain because besides the traditional "forward" strain it incorporates the "sidelong" strain into its imaginary part. By forward strain we meant the strain developed in the direction of the vector joining the two neighboring points used to measure strain. Instead of comparing the change in distance between two contiguous points, this concept describes the complete state of strains on an areola that surrounds a given point. The strain is given in terms of a total derivative and, as a consequence, the relative displacement between two arbitrary points of the strained plane is obtained through a line integral of the strain, along any path joining these points. In this approach, the strain of a plane is obtained by the division of two complex-valued quantities, associated with $2 \mathrm{D}$ vectors. The real part of the areolar strain is a radial strain while the imaginary part is either a circumferential strain derived from shear, or a rotation, see Fig. 1. Vector divisions are defined not only in the 2D vector space but also in the four-vector algebra and in the algebra of quaternions, which can probably be used to extend this strain concept to the three and four dimension spaces. Pinotsis [14] and Grigoriev and Alexin [15] have recently made progress in this field. Kirchhoff's four-order equation neglects transverse shear strain and is plagued by inconsistence between the order of the governing equation and the number of boundary conditions required. Most of the sixth-order theories, including the Mindlin-Reissner theory [7], require a correction factor to the transverse shear. The consideration of the rotation as a component of the strain,
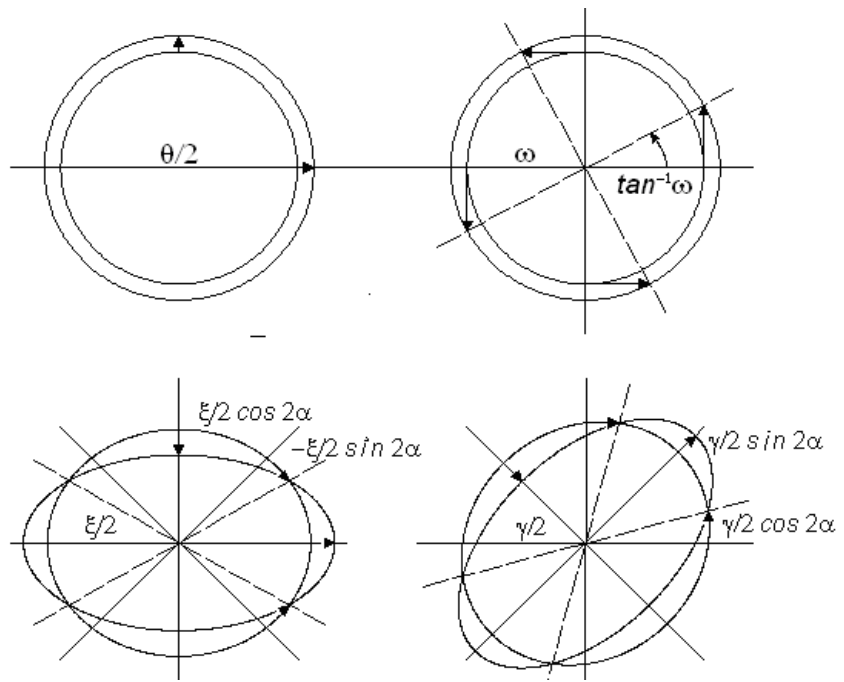

Figure 1: Fundamental modes of the plane strain. 
allowed expressing a compatible field of displacements and strains at the neighborhoods of the neutral plane of a plate. This field is then forced to comply with the boundary conditions. A sixth-order differential equation is obtained, which exactly gives the shearing forces, eliminating thus Kirchhoff's anomaly. As a result, only the shear and rotation of the areolas located at the neutral plane itself are sufficient to describe the displacement field through the thickness of the plate. This field is first verified by calculating the shear of beams and checking the results against those obtained through the 2D theory of elasticity. On the sequel, Kirchhoff's plate bending test is performed and verified that the total shear of the boundary of the plate matches the total normal load of the plate. The plate sag is decomposed into that due to pure bending and that due to shear only.

The stiffness of the plate is decomposed into the ones due to bending only and the other due to shear only.

\section{The areolar strain concept}

Let a region in the plane of the variables $z=x+i y$ and $\bar{z}=x-i y$ be mapped in a one-to-one manner onto the plane of the displacements $u(x, y)$ and $v(x, y)$ by means of the transformation $w(z, \bar{z})=u(x, y)+i v(x, y)$. The areolar strain is defined as the gradient of the vector field $w(z, \bar{z})$, through the Riemann derivative [13]:

$$
\begin{gathered}
\varepsilon=\lim _{z \rightarrow z_{0}} \frac{w-w_{0}}{z-z_{0}}=\frac{\frac{\partial w}{\partial z} d z+\frac{\partial w}{\partial z} d \bar{z}}{d z} \\
\varepsilon=\frac{\partial w}{\partial z}+\frac{\partial w}{\partial \bar{z}} e^{-i 2 \alpha}
\end{gathered}
$$

where the polar form has been used for the ratio

$$
\frac{d \bar{z}}{d z}=\frac{|d \bar{z}| e^{-i \alpha}}{|d z| e^{i \alpha}}=e^{-i 2 \alpha}
$$

The last expression presupposes that $z$ tends to $z_{0}$, maintaining the direction $\alpha$. Equation (1) is obtained making

with

$$
\begin{aligned}
& d u=\frac{\partial u}{\partial x} d x+\frac{\partial u}{\partial y} d y, d v=\frac{\partial v}{d x} d x+\frac{\partial v}{d y} d y \\
& d u+i d v=\frac{\partial w}{\partial z}(d x+i d y)+\frac{\partial w}{\partial \bar{z}}(d x-i d y)
\end{aligned}
$$

$$
\begin{aligned}
& 2 \frac{\partial w}{\partial z}=\left(\frac{\partial u}{\partial x}+\frac{\partial v}{\partial y}\right)+i\left(\frac{\partial v}{\partial x}-\frac{\partial u}{\partial y}\right)=\theta+i 2 \omega \\
& 2 \frac{\partial w}{\partial \bar{z}}=\left(\frac{\partial u}{\partial x}-\frac{\partial v}{\partial y}\right)+i\left(\frac{\partial v}{\partial x}+\frac{\partial u}{\partial y}\right)=\xi+i \gamma
\end{aligned}
$$

Equations (2) are the Kolosov-Wirtinger [13] derivatives. The areolar strain can hence be written in the form 


$$
\varepsilon=1 / 2(\theta+i 2 \omega)+1 / 2(\xi+i \gamma) e^{-2 i \alpha}
$$

When viewed in the polar form, see Fig. 1, the real part of the areolar strain represents a radial strain and the imaginary part represents either, a circumferential strain derived from shear or a rotation. In this conception, the plane strain is decomposed into two orthogonal complex strains shown at Fig. 1. The second complex term is the complex shear strain.

\section{Compatibility equations}

At reference [9] the following compatibility equations were obtained:

$$
\begin{aligned}
& \frac{\partial}{\partial x}(2 \omega-\gamma)+\frac{\partial}{\partial y}(\theta+\xi)=0 \\
& \frac{\partial}{\partial y}(2 \omega+\gamma)-\frac{\partial}{\partial x}(\theta-\xi)=0
\end{aligned}
$$

Saint-Venant's compatibility equation is obtained from these equations through elimination of the mode $\omega$, by applying a cross-differentiation followed by a subtraction.

\section{Polynomial expansion}

A polynomial expansion of the displacement field, which complies with the compatibility equations, will be derived with the purpose of representing the field of displacement in the neighborhoods of the neutral planes of beams and plates. Later on these displacements are forced to satisfy also the boundary conditions. As fairly well known, expansion of the displacement field $w(z, \bar{z})$ in Taylor's series from a fixed point $\mathrm{z}_{0}=0$ of the generalized complex plane, takes the form

$$
\begin{gathered}
w=w_{0}+\frac{\partial w}{\partial z} z+\frac{\partial w}{\partial \bar{z}} \bar{z}+\frac{\partial^{2} w}{\partial z \partial z} \frac{z^{2}}{2}+\frac{\partial^{2} w}{\partial z \partial \bar{z}} \frac{z \bar{z}}{2}+ \\
+\frac{\partial^{2} w}{\partial \bar{z} \partial z} \frac{\overline{z z}}{2}+\frac{\partial^{2} w}{\partial \bar{z} \partial \bar{z}} \frac{\bar{z}^{2}}{2}+\frac{\partial^{3} w}{\partial z^{3}} \frac{z^{3}}{6}+\ldots
\end{gathered}
$$

where all derivatives are calculated at $z_{0}$. The derivatives of the displacements are calculated in terms of the orthogonal strain components, in accordance with equation (2), and submitted to satisfy the compatibility equations (4), by the elimination of mode $\xi$, giving

$$
\begin{aligned}
& \frac{\partial \xi}{\partial x}=-\frac{\partial \gamma}{\partial y}-2 \frac{\partial \omega}{\partial y}+\frac{\partial \theta}{\partial x} \\
& \frac{\partial \xi}{\partial y}=-\frac{\partial \theta}{\partial y}+\frac{\partial \gamma}{\partial x}-2 \frac{\partial \omega}{\partial x}
\end{aligned}
$$

Modes $\theta$ and $\omega$ are harmonic functions and the following relations hold true 


$$
\begin{aligned}
& \frac{\partial^{2} \omega}{\partial y^{2}}=-\frac{\partial^{2} \omega}{\partial x^{2}} \\
& \frac{\partial^{2} \theta}{\partial y^{2}}=-\frac{\partial^{2} \theta}{\partial x^{2}}
\end{aligned}
$$

The compatible derivatives of the polynomial expansion are then reduced to

$$
\begin{gathered}
\frac{\partial^{2} w}{\partial z^{2}}=\frac{1}{4}\left[\frac{\partial}{\partial y}(2 \omega-i \theta)+\frac{\partial}{\partial x}(\theta+2 i \omega)\right] \\
\frac{\partial^{2} w}{\partial \bar{z}^{2}}=\frac{1}{4} \frac{\partial \theta}{\partial x}-\frac{1}{2} \frac{\partial}{\partial y}(\gamma+\omega)+\frac{i}{4}\left[-2 \frac{\partial \omega}{\partial x}+\frac{\partial}{\partial y}(\gamma-\theta)\right] \\
\frac{\partial^{2} w}{\partial z \partial \bar{z}}=\frac{1}{4}\left[\frac{\partial}{\partial x}(\theta+i 2 \omega)-\frac{\partial}{\partial y}(2 \omega-i \theta)\right] \\
\frac{\partial^{3} w}{\partial z^{3}}=\frac{1}{4}\left(\frac{\partial^{2} \theta}{\partial x^{2}}+2 \frac{\partial^{2} \omega}{\partial x \partial y}\right)-\frac{i}{4}\left(\frac{\partial^{2} \theta}{\partial x \partial y}-2 \frac{\partial^{2} \omega}{\partial x^{2}}\right) \\
\frac{\partial^{3} w}{\partial z \partial \bar{z}^{2}}=\frac{1}{4}\left[\frac{\partial^{2} w}{\partial x^{2}}(\theta+i 2 \omega)-\frac{\partial^{2}}{\partial x \partial y}(2 \omega-i \theta)\right] \\
\frac{\partial^{3} w}{\partial \bar{z}^{3}}=-\frac{3}{8} \frac{\partial^{2} \gamma}{\partial x \partial y}+\frac{i}{8}\left(\frac{\partial^{2} \gamma}{\partial x^{2}}-2 \frac{\partial^{2} \gamma}{\partial y^{2}}\right)
\end{gathered}
$$

Substitution of these derivatives at equation (5) and elimination of the polynomials in $x$ lead to the following expression for the field of displacements in the $X$ direction of the (vertical) plane $X-Y$

$$
U(x, y)=y\left[\frac{1}{2} \gamma(x)-\omega(x)\right]-y^{3}\left[f(x)+\frac{\partial^{2} \gamma(x)}{\partial x^{2}}-8 \frac{\partial^{2} \omega(x)}{\partial x^{2}}\right]
$$

where $f(x)$ represents a sum of derivatives in y. Now, assuming that all planes parallel to the neutral plane will remain parallel after deformation, the shear and the rotation of a point of the plane $X-Y$, in the neighborhoods of the neutral plane, will be given by

$$
\begin{aligned}
& \Gamma(x, y]=\frac{\partial v(x)}{\partial x}+\frac{\partial U(x, y)}{\partial y} \\
& \Omega(x, y)=\frac{1}{2}\left(\frac{\partial v(x)}{\partial x}-\frac{\partial U(x, y)}{\partial y}\right)
\end{aligned}
$$

where $v$ is the displacement in the $\mathrm{Y}$ direction. Hence after equation (15)

$$
\begin{gathered}
\Gamma(x, y)=\frac{\gamma(x)}{2}-\omega(x)+\frac{\partial v(x)}{d x}+\frac{1}{16} y^{2}\left[f(x)-\frac{\partial^{2} \gamma(x)}{\partial x^{2}}+8 \frac{\partial^{2} \omega(x)}{\partial x^{2}}\right] \\
\Omega(x, y)=\frac{1}{2} \omega(x)-\frac{1}{4} \gamma(x)+\frac{1}{2} \frac{\partial v}{\partial x}+\frac{y^{2}}{32}\left[-f(x)+\frac{\partial^{2} \gamma(x)}{\partial x^{2}}-8 \frac{\partial^{2} \omega(x)}{\partial x^{2}}\right]
\end{gathered}
$$


Imposing zero-shear at the top and bottom of the beam and equating the rotation $\Omega(x, 0)$ to the rotation of the areola located at the neutral plane, through equations

$$
\begin{aligned}
& \Gamma\left(x, \frac{h}{2}\right)=0 \\
& \Omega(x, 0)=\omega(x)
\end{aligned}
$$

equations (17) give

$$
\begin{gathered}
\frac{\partial v(x)}{\partial x}=\omega(x)+\frac{1}{2} \gamma(x) \\
f(x)=-\frac{64 \gamma(x)}{h^{2}}+\frac{\partial^{2} \gamma}{\partial x^{2}}-8 \frac{\partial^{2} \omega}{\partial x^{2}}
\end{gathered}
$$

Equation (19) shows that the rotation of the neutral plane does not follow the rotation of the areola located on the neutral plane; being increased by the rotation resulted from shear. Substitution of equation (20) at equation (15) results

$$
U(x, y)=-y \omega(x)+\frac{y}{6}\left[\left(3-\frac{8 y^{2}}{h^{2}}\right) \gamma(x)\right.
$$

and substitution of this equation and equation (19) at equations (16) completes the set of equations required to describe a compatible field of displacements and strains at the neighborhoods of the neutral plane, through the shear and rotation of the areolas located at the neutral plane itself. Axial strain and displacement are not encompassed. Shear and rotation in the neighborhoods of the neutral plane are hence given by

$$
\begin{gathered}
\Gamma(x, y)=\left(1-\frac{4 y^{2}}{h^{2}}\right) \gamma(x) \\
\Omega(x, y)=\omega(x)+\frac{2 y^{2} \gamma(x)}{h^{2}}
\end{gathered}
$$

\section{Beams}

In order to test the above function, it is first applied to beams. Using Hooke's law, the bending stress at a section, according with equation (21), is given by

$$
\sigma_{x}(x, y)=y E\left[-\frac{\partial \omega(x)}{\partial x}+\left(\frac{3}{6}-\frac{8 y^{2}}{6 h^{2}}\right) \frac{\partial \gamma(x)}{\partial x}\right]
$$

For a rectangular beam with a high $h$ and unit width, the moments and shearing forces are

$$
\begin{gathered}
M_{x}(x)=\int_{-h / 2}^{h / 2} \sigma_{x}(x, y) y d y=-\frac{E h^{3}}{12}\left(\frac{\partial \omega}{\partial x}-\frac{3}{10} \frac{\partial \gamma}{\partial x}\right) \\
Q_{x}(x)=\mu \int_{-h / 2}^{h / 2} \Gamma(x, y) d y=\frac{2}{3} h \mu \gamma(x)
\end{gathered}
$$

and the equilibrium equation for distributed load

$$
q(x)=\frac{\partial^{2} M_{x}}{\partial x^{2}}=-\frac{E h^{3}}{12}\left(\frac{\partial^{3} \omega}{\partial x^{3}}-\frac{3}{10} \frac{\partial^{3} \gamma}{\partial x^{3}}\right)
$$




\subsection{Simply supported with uniformly distributed load}

Let $L$ be the span of a simple supported beam with a uniformly distributed load $q$, then

$$
\begin{gathered}
Q(x)=q(L / 2-x), M(x)=\frac{q\left(L x-x^{2}\right)}{2} \\
\gamma(x)=\frac{3 q(L / 2-x)}{2 h \mu}
\end{gathered}
$$

Integrating equation (27) gives

$$
\omega(x)=-\frac{3 L q x^{2}}{h^{3} E}+\frac{2 q x^{3}}{h^{3} E}-\frac{9 q x}{20 h \mu}+A_{1}
$$

Substitution on $\frac{\partial v(x)}{\partial x}=\omega(x)+\frac{1}{2} \gamma(x)$ and integrating results

$$
v(x)=-\frac{L q x^{3}}{h^{3} E}+\frac{q x^{4}}{2 h^{3} E}+\frac{3 L q x}{8 h \mu}-\frac{3 q x^{2}}{5 h \mu}+A_{1} x+A_{2}
$$

The constants of integration represent a rigid body motion and therefore, for simple-supported beams they are null. At the center, the deflection reads

$$
v(L / 2)=-\frac{5 L^{4} q}{32 h^{3} E}-\frac{3 L^{2} q}{20 h \mu}
$$

The above results are in agreement with solutions obtained through the twodimensional theory of elasticity. See for example Timoshenko and Goodier [1]. For frames, the practice is to force the neutral planes of the beams to conserve their relative angles at the junctions. In the theory above presented, the same rotation of all beams at the junction will not be achieved. Nevertheless, at some distance from the junction, the rotations tend to the theoretical values, according to SaintVenant's principle.

\section{Plate bending}

Similarly to equation (21), at a positive distance $\zeta$ from the (now horizontal) neutral plane $X-Y$, the displacements will be taken in the form

$$
\begin{aligned}
& u(x, y, \zeta)=-\zeta \omega_{x}(x, y)+\left(\frac{\zeta}{2}-\frac{8 \zeta^{3}}{6 h^{2}}\right) \gamma_{x}(x, y) \\
& v(x, y, \zeta)=-\zeta \omega_{y}(x, y)+\left(\frac{\zeta}{2}-\frac{8 \zeta^{3}}{6 h^{2}}\right) \gamma_{y}(x, y)
\end{aligned}
$$

Considering that the plate is submitted to a plane stress state, the traction at a (vertical) section normal to the $X$ axis, is given by equation (14) of reference [9]

$$
T_{x}=(\lambda *+\mu) \Theta(x, y, \zeta)-\mu[\Xi(x, y, \zeta)+i \Gamma(x, y, \zeta)] e^{-2 i(\pi / 2)}
$$

with $\lambda^{*}=\frac{2 \lambda \mu}{\lambda+2 \mu}$, where $\lambda$ and $\mu$ are Lame's elastic constants, and 


$$
\Theta(x, y, \zeta)=\frac{\partial u}{\partial x}+\frac{\partial v}{\partial y}, \quad \Xi(x, y, \zeta)=\frac{\partial u}{\partial x}-\frac{\partial v}{\partial y}, \quad \Gamma(x, y, \zeta)=\frac{\partial v}{\partial x}+\frac{\partial u}{\partial y}
$$

are areolar components of the strain, given at equation (2). Taking the real and imaginary parts of the traction, the normal and tangential stresses will read

$$
\begin{gathered}
\sigma_{x}=\frac{\zeta \mu}{3 h^{2}(\lambda+2 \mu)}\left\{\lambda\left(3 h^{2}-8 \zeta^{2}\right) \frac{\partial \gamma_{y}}{\partial y}-6 h^{2} \lambda \frac{\partial \omega_{y}}{\partial y}+\right. \\
\left.2(\lambda+\mu)\left[\left(3 h^{2}-8 \zeta^{2}\right) \frac{\partial \gamma_{x}}{\partial x}-6 h^{2} \frac{\partial \omega_{x}}{\partial x}\right]\right\} \\
\tau_{x y}=\zeta \mu\left[\frac{\left(3 h^{2}-8 \zeta^{2}\right)}{6 h^{2}}\left(\frac{\partial \gamma_{x}}{\partial y}+\frac{\partial \gamma_{y}}{\partial x}\right)+\left(\frac{\partial \omega_{x}}{\partial y}+\frac{\partial \omega_{y}}{\partial x}\right)\right]
\end{gathered}
$$

and, by symmetry

$$
\begin{aligned}
\sigma_{y}=\frac{\zeta \mu}{3 h^{2}(\lambda+2 \mu)}\left\{\left(3 h^{2}-8 \zeta^{2}\right) \lambda \frac{\partial \gamma_{x}}{\partial x}-6 h^{2} \lambda \frac{\partial \omega_{x}}{\partial x}+\right. \\
\left.2(\lambda+\mu)\left[\left(3 h^{2}-8 \zeta^{2}\right) \frac{\partial \gamma_{y}}{\partial y}-6 h^{2} \frac{\partial \omega_{y}}{\partial y}\right]\right\}
\end{aligned}
$$

For the rotations of the areolas situated at the neutral plane we take, similarly to equation (19).

$$
\begin{aligned}
& \omega_{x}(x, y)=\frac{\partial w}{\partial x}-\frac{1}{2} \gamma_{x}(x, y) \\
& \omega_{y}(x, y)=\frac{\partial w}{\partial y}-\frac{1}{2} \gamma_{y}(x, y)
\end{aligned}
$$

and substitute at the above stress equations. The first two equilibrium equations of an infinitesimal solid element are then

$$
\begin{aligned}
& \frac{\partial \sigma_{x}(x, y, \zeta)}{\partial x}+\frac{\partial \tau_{x y}(x, y, \zeta)}{\partial y}+\frac{\partial}{\partial \zeta}\left[\mu\left(1-\frac{4 \zeta^{2}}{h^{2}}\right) \gamma_{x}(x, y)\right]=0 \\
& \frac{\partial \sigma_{y}(x, y, \zeta)}{\partial y}+\frac{\partial \tau_{x y}(x, y, \zeta)}{\partial x}+\frac{\partial}{\partial \zeta}\left[\mu\left(1-\frac{4 \zeta^{2}}{h^{2}}\right) \gamma_{y}(x, y)\right]=0
\end{aligned}
$$

This is a difficult to solve system of second order differential equations of the unknowns variables $\gamma_{x}$ and $\gamma_{y}$. However for our purposes, we can use a simplified form, by disregarding the second derivatives of $\gamma_{x}$ and $\gamma_{y}$, and use equation (26), which results in the classical equation for shear in plates

$$
\begin{aligned}
& \gamma_{x}(x, y)=-\frac{h^{2}(\lambda+\mu)}{2(\lambda+2 \mu)} \frac{\partial}{\partial x}\left(\frac{\partial^{2} w}{\partial x^{2}}+\frac{\partial^{2} w}{\partial y^{2}}\right) \\
& \gamma_{y}(x, y)=-\frac{h^{2}(\lambda+\mu)}{2(\lambda+2 \mu)} \frac{\partial}{\partial y}\left(\frac{\partial^{2} w}{\partial x^{2}}+\frac{\partial^{2} w}{\partial y^{2}}\right)
\end{aligned}
$$

Let $h$ be the thickness of the plate. Calculation of the forces for a unit width strip, results 


$$
\begin{gathered}
M_{x}=\int_{-h / 2}^{h / 2} \sigma_{x} \zeta d \zeta=-\frac{h^{3} \mu}{6(\lambda+2 \mu)}\left[2(\lambda+\mu) \frac{\partial^{2} w}{\partial x^{2}}+\lambda \frac{\partial^{2} w}{\partial y^{2}}\right]+ \\
-\frac{h^{5} \mu(\lambda+\mu)}{15(\lambda+2 \mu)^{2}}\left[2(\lambda+\mu) \frac{\partial^{4} w}{\partial x^{4}}+(3 \lambda+2 \mu) \frac{\partial^{4} w}{\partial x^{2} \partial y^{2}}+\lambda \frac{\partial^{4} w}{\partial y^{4}}\right] \\
M_{y}=\int_{-h / 2}^{h / 2} \sigma_{y} \zeta d \zeta=-\frac{h^{3} \mu}{6(\lambda+2 \mu)}\left[2(\lambda+\mu) \frac{\partial^{2} w}{\partial y^{2}}+\lambda \frac{\partial^{2} w}{\partial x^{2}}\right]+ \\
-\frac{h^{5} \mu(\lambda+\mu)}{15(\lambda+2 \mu)^{2}}\left[2(\lambda+\mu) \frac{\partial^{4} w}{\partial y^{4}}+(3 \lambda+2 \mu) \frac{\partial^{4} w}{\partial x^{2} \partial y^{2}}+\lambda \frac{\partial^{4} w}{\partial x^{4}}\right] \\
M_{x y}=-M_{y x}=\int_{-h / 2}^{h / 2} \tau_{x y} \zeta d \zeta= \\
\frac{h^{3} \mu}{30(\lambda+2 \mu)} \frac{\partial^{2} w}{\partial x \partial y}\left[5(\lambda+2 \mu)+2 h^{2}(\lambda+\mu)\left(\frac{\partial^{2} w}{\partial x^{2}}+\frac{\partial^{2} w}{\partial y^{2}}\right)\right]
\end{gathered}
$$

Solving the following equilibrium equations for $Q_{x}$ and $Q_{y}$

$$
\begin{aligned}
& \frac{\partial M_{x y}}{\partial x}-\frac{\partial M_{y}}{\partial y}+Q_{y}=0 \\
& \frac{\partial M_{x y}}{\partial y}+\frac{\partial M_{x}}{\partial x}-Q_{x}=0
\end{aligned}
$$

gives the shearing forces

$$
\begin{aligned}
& Q_{x}=-\frac{h^{3} \mu(\lambda+\mu)}{3(\lambda+2 \mu)} \frac{\partial \Delta}{\partial x}-\frac{2 h^{5} \mu(\lambda+\mu)^{2}}{15(\lambda+2 \mu)^{2}} \frac{\partial \Delta \Delta}{\partial x} \\
& Q_{y}=-\frac{h^{3} \mu(\lambda+\mu)}{3(\lambda+2 \mu)} \frac{\partial \Delta}{\partial y}-\frac{2 h^{5} \mu(\lambda+\mu)^{2}}{15(\lambda+2 \mu)^{2}} \frac{\partial \Delta \Delta}{\partial y}
\end{aligned}
$$

where $\Delta$ stands for the Laplacian operator. Substitutions of the shearing forces into third equilibrium equation

$$
\frac{\partial Q_{x}}{\partial x}+\frac{\partial Q_{y}}{\partial y}+q(x, y)=0
$$

gives

$$
\begin{aligned}
q(x, y)= & \frac{h^{3}(\lambda+\mu) \mu}{3(\lambda+2 \mu)}\left(\frac{\partial^{4} w}{\partial x^{4}}+2 \frac{\partial^{4} w}{\partial x^{2} \partial y^{2}}+\frac{\partial^{4} w}{\partial y^{4}}\right)+ \\
& \frac{2 h^{5}(\lambda+\mu)^{2} \mu}{15(\lambda+2 \mu)^{2}}\left(\frac{\partial^{6} w}{\partial x^{6}}+3 \frac{\partial^{6} w}{\partial x^{2} \partial y^{4}}+3 \frac{\partial^{6} w}{\partial x^{4} \partial y^{2}}+\frac{\partial^{6} w}{\partial y^{6}}\right)
\end{aligned}
$$

As $\lambda=\frac{E v}{(1+v)(1-2 v)}$ and $\mu=\frac{E}{2(1+v)}$ this equation can be written in the compact form

$$
q(x, y)=D_{\omega} \Delta \Delta w(x, y)+D_{\gamma} \Delta \Delta \Delta w(x, y)
$$

The flexural and shearing rigidities read 


$$
\begin{gathered}
D_{\omega}=\frac{E h^{3}}{12\left(1-v^{2}\right)} \\
D_{\gamma}=\frac{E h^{5}}{60(1-v)^{2}(1+v)}
\end{gathered}
$$

\subsection{Kirchhoff's plate bending test}

Following Chapter 5 from Timoshenko's and Woinowsky-Krieger book [2], let

$$
\begin{aligned}
& q=q_{0} \sin \left(\frac{\pi x}{a}\right) \sin \left(\frac{\pi y}{b}\right) \\
& w=c \sin \left(\frac{\pi x}{a}\right) \sin \left(\frac{\pi y}{b}\right)
\end{aligned}
$$

Solving equation (48) for c, results

$$
c=\frac{15 a^{6} b^{6} q_{0}(\lambda+2 \mu)^{2}}{\left(a^{2}+b^{2}\right)^{2} h^{3} \pi^{4} \mu(\lambda+\mu)\left[-2\left(a^{2}+b^{2}\right) h^{2} \pi^{2}(\lambda+\mu)+5 a^{2} b^{2}(\lambda+2 \mu)\right]}
$$

If we take into consideration the second term of the denominator only, we recognize the bending sag

$$
c_{\omega}=\frac{3 a^{4} b^{4} q_{0}(\lambda+2 \mu)}{\left(a^{2}+b^{2}\right)^{2} h^{3} \pi^{4} \mu(\lambda+\mu)}
$$

and the shear sag is obtained through

$$
c_{\gamma}=c-c_{\omega}=\frac{6 a^{4} b^{4} q_{0}(\lambda+2 \mu)}{\left(a^{2}+b^{2}\right) h \pi^{2} \mu\left[-2\left(a^{2}+b^{2}\right) h^{2} \pi^{2}(\lambda+\mu)+5 a^{2} b^{2}(\lambda+2 \mu)\right]}
$$

Substitution of $w$ on equations (46) simple results

$$
\begin{aligned}
& Q_{x}=\frac{a b^{2} q_{0}}{\pi\left(a^{2}+b^{2}\right)} \cos \left(\frac{\pi x}{a}\right) \sin \left(\frac{\pi y}{b}\right) \\
& Q_{y}=\frac{a^{2} b q_{0}}{\pi\left(a^{2}+b^{2}\right)} \sin \left(\frac{\pi x}{a}\right) \cos \left(\frac{\pi y}{b}\right)
\end{aligned}
$$

and the sum of boundary transverse shears exactly match the applied load $P$.

$$
\begin{gathered}
P=\int_{0}^{a} \int_{0}^{b} q_{0} \sin \left(\frac{\pi x}{a}\right) \sin \left(\frac{\pi y}{b}\right) d y d x=\frac{4 a b q_{0}}{\pi^{2}} \\
\int_{0}^{b}\left(Q_{x}(0, y)-Q_{x}(a, y) \mathrm{d} y+\int_{0}^{a}\left(Q_{y}(x, 0)-Q_{y}(x, b)\right) \mathrm{d} x=\frac{4 a b q_{0}}{\pi^{2}}\right.
\end{gathered}
$$

Putting $q_{0}=1, a=1, b=a, h=a / 10, \lambda=1, \mu=1$, results $c_{\omega}=11.54923$ and $c_{\gamma}=0.6417$.The bending sag exactly matches the classical theory. 


\section{Conclusion}

Kirchhoff's bi-harmonic equation neglects transverse shear strain and is plagued by inconsistence between the order of the governing equation and the number of boundary conditions required. Most of the sixth-order theories require a correction factor to the transverse shear. Several distributions of in plane displacements through the thickness of the plate have being tried. See references [3-9]. Most of them suffered from the imperfection of violating the compatibility conditions of deformation of elastic solids. In the present method, the compatibility of the deformed field was attained at the neighborhoods of the neutral plane through a Taylor expansion, whose derivatives are compelled to obey the correct equations of compatibility derived by the writer. The inclusion of the rotation as a component of the strain, introduced by the writer, allowed expressing compatible fields of displacements and strains at the neighbourhoods of the neutral plane, considering only the shear and the rotation of the areolas located at the neutral plane itself. The parabolic distribution of transverse shear proved to lead to more amenable to solve equations for beams as well as for plates. This tri-harmonic equation allows the prescription of complete boundary conditions.

\section{References}

[1] Timoshenko and Goodier, Theory of Elasticity, Chapter 3, 2nd Edition, McGraw-Hill, 1951.

[2] Timoshenko and Woinowsky-Krieger, Theory of Plates and Shells, Chapters 4 and 5, 2nd Edition, McGraw-Hill, 1959.

[3] Fan Yeli and Ling Fangyong, An Analytical Solution of Rectangular Laminated Plates by Higher-order Theory, Applied Mathematics and Mechanics, Vol. 19, No. 8, 1998.

[4] Guangyu Shi and Voyiadjis, G.Z., A Sixth-Order Theory of Shear Deformable Beams, ASME Journal of Applied Mechanics, Vol. 78, 2011.

[5] Murthy, M.V.V., An Improved Transverse Shear Deformation Theory for Laminated Anisotropic Plates, NASA Technical Paper 1903, 1981.

[6] Reddy, J.N., A generalization of two-dimensional theories of laminated composite plates', Communications in Applied Numerical Methods, 3, 173-180, 1987.

[7] Reissner, E., The effect of transverse shear deformation on the bending of elastic plates, ASME Journal of Applied Mechanics, Vol. 12, pp. A68-77, 1945.

[8] Wang, C. M., Lim, G. T., Reddy, J. N, and Lee, K. H, Relationships between bending solutions of Reissner and Mindlin plate theories, Engineering Structures, vol. 23, pp. 838-849, 2001.

[9] Kotchergenko, I.D., The Areolar Strain Concept Applied to Elasticity, WIT Transactions on Modelling and Simulation, Vol. 46, 2007, WIT Press, (Open Access).

[10] Kotchergenko, I.D., The Areolar Strain Concept, Mechanics of Solids, Structures and Fluids, Volume 12, ASME, IMECE-2008. 
[11] Kotchergenko, I.D., The Areolar Strain Approach for grazing waves, WIT Transactions on Modelling and Simulation, Vol. 55, 2013, WIT Press, (Open Access).

[12] Kotchergenko, I.D., Kolosov-Mushkhelishvili Formulas Revisited, $11^{\text {th }}$ International Conference on Fracture, Turin, March 2005.

[13] Mitrinovic, D.S. and Keckic, J.D., From the History of Nonanalitic Functions, Série: Mathématiques e Physique, No. 274-371, Publications de La Faculté D'Electrotechnique de L'Université à Belgrade, 1969, (Open Access).

[14] Pinotsis, D.A., Quaternionic Analysis, Elliptic Problems and a Physical Application of the Dbar Formalism, Advances in Applied Clifford Algebras, Verlag, 2010.

[15] Grigoriev, Yu.M. and Alexin, V.V., Quaternionic Method for Boundary Elements, Sibirsky Journal, Tom II, No. 1, 1999 (Russian). 